\title{
First Integrals and Hamiltonians of Some Classes of ODEs of Maximal Symmetry
}

\author{
J. C. Ndogmo \\ Department of Mathematics and Applied Mathematics, University of Venda, P/B X5050, Thohoyandou, Limpopo 0950, South Africa \\ Correspondence should be addressed to J. C. Ndogmo; ndogmoj@yahoo.com
}

Received 5 December 2016; Revised 12 January 2017; Accepted 22 January 2017; Published 14 February 2017

Academic Editor: Peter G. L. Leach

Copyright (C) 2017 J. C. Ndogmo. This is an open access article distributed under the Creative Commons Attribution License, which permits unrestricted use, distribution, and reproduction in any medium, provided the original work is properly cited.

Complete sets of linearly independent first integrals are found for the most general form of linear equations of maximal symmetry algebra of order ranging from two to eight. The corresponding Hamiltonian systems are constructed and it is shown that their general solutions can also be found by a simple superposition formula from the solutions of a scalar second-order source equation.

\section{Introduction}

An important class of linear ordinary differential equations (LODEs) consists of those equations having a symmetry algebra of maximal dimension. This is partly due to the simple characterization of such a class of equations [1,2], according to which they are precisely the iterative equations, and equivalently they can be reduced by a point transformation to the canonical form $y^{(n)}=0$.

Despite the fact that linear equations are the simplest types of differential equations, they are far from being completely understood and yet they frequently appear in the study of all other types of equations and in particular as canonical or reduced form of nonlinear or partial differential equations with more complex structures.

Linear equations with maximal symmetry algebras have been studied in some recent papers [3-6], but in all such papers the emphasis has been placed more on the coefficient characterization and the elementary symmetry or the transformation properties of these equations. Nœther's theorem $[7,8]$ is a powerful tool that allows us to associate each variational symmetry of the equation with a conservation law, and, in the case of ordinary differential equations (ODEs), these conservation laws correspond to first integrals which in this instance are true constants of motion. The role of these first integrals is often crucial in the study of solutions and properties of differential equations, including questions related to stability $[9,10]$ or integrability $[11-14]$. The so-called first integral method for finding exact solutions of nonlinear partial differential equations has also been successfully applied to a wide range of equations modeling various phenomena in physics (see [15] and the references therein).

In this paper, we obtain a complete set of linearly independent first integrals for all linear equations of maximal symmetry of order $n$ such that $2 \leq n \leq 8$. These first integrals are obtained for the most general form of the equations in which they depend on an arbitrary function through an application of Nœther's theorem. We also give the Hamiltonian formulation for the corresponding class of scalar equations and show amongst others that their general solution can also be obtained by a simple superposition formula from those of a scalar second-order source equation.

\section{Basic Properties of Linear Iterative Equations}

Let $r \neq 0$ and $s$ be two functions of the variable $x$ and consider the differential operator $\Psi=r(d / d x)+s$. Denote by $\Delta_{n}[y]=$ 0 a LODE of order $n$ in the dependent variable $y=y(x)$. Then this equation is said to be iterative if up to a normalizing factor one has $\Delta_{n}[y]=\Psi^{n}[y]$, where

$$
\begin{aligned}
& \Psi^{n}[y]=\Psi^{n-1}[\Psi[y]], \\
& \text { for } n \geq 1 \text { with } \Psi^{0}=I, \Psi^{1}=\Psi
\end{aligned}
$$


and where $I$ is the identity operator. A LODE $\Delta_{n}[y]=0$ has a maximal symmetry algebra if and only if it is iterative [1] or, equivalently $[1,2]$, if and only if it can be reduced by a point transformation to the canonical form $y^{(n)}=0$. By the transformation $w=y \exp \left((1 / n) \int_{x_{0}}^{x} B_{n-1}(v) d v\right)$, where $B_{n-1}(z)$ is the coefficient of the term of order $n-1$, any given LODE may be put into the form

$$
y^{(n)}+\sum_{i=0}^{n-2} A_{n}^{n-i}(x) y^{(i)}=0
$$

which is referred to as the normal form of the equation. For linear iterative equations in the latter form, $n$ linearly independent solutions can be found in the form

$$
s_{k}=u^{n-k-1} v^{k}, \quad 0 \leq k \leq n-1, n \geq 2,
$$

for some functions $u$ and $v$ of the independent variable $x$. For a given function $F=F(t)$, we shall also use the notation $F_{t}, F_{t t}$, and so forth for $d F / d t, d^{2} F / d t^{2}$, and so forth, but where convenient we shall also denote $d F / d t$ simply by $F^{\prime}$.

It follows in particular from (3) that $u$ and $v$ are two linearly independent solutions of the second-order so-called source equation:

$$
y_{x x}+\mathfrak{q} y=0, \quad \mathfrak{q}=A_{2}^{2} .
$$

In particular, we have

$$
\mathfrak{q}=w\left(u^{\prime}, v^{\prime}\right)
$$

Thanks to Abel's identity, the Wronskian $w(u, v)=\operatorname{det}\left(\begin{array}{cc}u & v \\ u^{\prime} & v^{\prime}\end{array}\right)$ of two solutions $u$ and $v$ of a LODE in the form (2) is a constant and may be normalized to one as we shall do by convention.

By a result of Lie [16], all equations of order $n \geq 2$ reducible by a point transformation to the canonical form $y^{(n)}=0$ have a symmetry algebra $\mathfrak{g}_{n}$ of maximal dimension 8 for $n=2$ and $n+4$ for $n \geq 3$. Owing to the linear property, the $n+1$ vector fields

$$
\begin{aligned}
& V_{k}=s_{k} \partial_{y}=u^{n-k-1} v^{k} \partial_{y}, \quad 0 \leq k \leq n-1, \\
& W_{y}=y \partial_{y}
\end{aligned}
$$

are symmetries of any iterative equation of the form (2). The three additional ones,

$$
\begin{aligned}
& F_{n}=u^{2} \partial_{x}+(n-1) u u_{x} y \partial_{y}, \\
& G_{n}=2 u v \partial_{x}+(n-1)\left(u v_{x}+u_{x} v\right) y \partial_{y}, \\
& H_{n}=-v^{2} \partial_{x}-(n-1) v v_{x} y \partial_{y},
\end{aligned}
$$

obtained in [1], are also generators of the Lie symmetry algebra $\mathfrak{g}_{n}$ of (2) in the case of equations of maximal symmetry. These $n+4$ symmetries form a basis of $\mathfrak{g}_{n}$ for $n \geq 3$. For $n=2$, a basis of $\mathfrak{g}_{n}$ consists of the same $n+4$ symmetry generators together with the two non-Cartan symmetries given by

$$
\begin{aligned}
& C_{1}=y u \partial_{x}+y^{2} u^{\prime} \partial_{y}, \\
& C_{2}=y v \partial_{x}+y^{2} v^{\prime} \partial_{y} .
\end{aligned}
$$

Recall that, in (6a)-(6f), $u$ and $v$ are assumed to be two linearly independent solutions of the source equation (4). The symmetry generators $V_{k}$ in (6a) associated with each solution $s_{k}$ are often called solution symmetries and generate the abelian Lie algebra $\mathscr{A}_{n}$, while $W_{y}$ is referred to as the homogeneity symmetry. Denoting by $\mathbf{v}^{\mathrm{Q}}=\mathrm{Q} \partial_{y}$ the characteristic form of a vector field $\mathbf{v}$ with characteristic $Q$, it follows that the characteristic forms of the infinitesimal generators listed in (6a)-(6f) are given by

$$
\begin{aligned}
V_{k}^{\mathrm{Q}} & =V_{k}, \quad 0 \leq k \leq n-1, \\
F_{n}^{\mathrm{Q}} & =\left[(n-1) u u_{x} y-u^{2} y_{x}\right] \partial_{y}, \\
H_{n}^{\mathrm{Q}} & =\left[(n-1) v v_{x} y+v^{2} y_{x}\right] \partial_{y}, \\
W_{y}^{\mathrm{Q}} & =W_{y}, \\
C_{1}^{\mathrm{Q}} & =\left(y^{2} u^{\prime}-u y y^{\prime}\right) \partial_{y}, \\
C_{2}^{\mathrm{Q}} & =\left(y^{2} v^{\prime}-v y y^{\prime}\right) \partial_{y}, \\
G_{n}^{\mathrm{Q}} & =\left[(n-1)(u v)^{\prime} y-2 u v y_{x}\right] \partial_{y}
\end{aligned}
$$

In the actual case of LODEs, the characteristic $Q$ of $\mathbf{v}$ has the simpler expression $Q=\mathbf{v}(y)-\mathbf{v}(x) y_{x}$.

\section{Variational Symmetries and First Integrals}

Suppose that the equation $\Delta_{n}[y]=0$ is the Euler-Lagrange equation of some variational problem corresponding to a Lagrangian $L$. Let

$$
\mathbf{v}=\xi(x, y) \partial_{x}+\phi(x, y) \partial_{y}
$$

be a symmetry generator with characteristic function $Q$ of the equation and set

$$
\begin{aligned}
& \mathcal{S}(\mathbf{v})=\mathbf{v}^{[n]}(L)+L \operatorname{div} \xi, \quad(\text { for } n \text { even }) \\
& \mathscr{D}(\mathbf{v})=D_{\Delta}^{*}(Q)+D_{\mathrm{Q}}^{*}(\Delta)
\end{aligned}
$$

where $\mathbf{v}^{[n]}$ is the $n$th prolongation of the vector field $\mathbf{v}$ and $D_{P}^{*}$ is the adjoint Fréchet derivative of the differential function $P=P[y]$. The vanishing of $\mathbf{v}$ under these two operators,

$$
\begin{aligned}
& \mathcal{S}(\mathbf{v})=0, \\
& \mathscr{D}(\mathbf{v})=0,
\end{aligned}
$$

represents some key concepts. Indeed, in the sense of [7], $\mathbf{v}$ is called a variational symmetry for $\Delta_{n}[y]=0$ if it satisfies (10) and is called a divergence symmetry if it satisfies (11). The 
latter condition is equivalent to $\delta(\mathbf{v})=\operatorname{div} B$ for a certain differential function $B=B[y]$. Divergence symmetries are thus a more relaxed form of variational symmetries, and they also give rise to conservation laws and first integral as variational symmetries do by Nœther's theorem. Divergence symmetries satisfy the same condition required on generalized symmetries to be variational symmetries, and, in a more modern language [17], these divergence symmetries are simply called variational symmetries. The characteristic form of a conservation law associated with each divergence symmetry has the form $Q \Delta_{n}[y]=\operatorname{div} P$, for a certain differential function $P=P[y]$. In the actual case of an ODE, we have

$$
Q \Delta_{n}[y]=D P, \quad \text { where } D=\frac{d}{d x}
$$

is the total differential operator. Thus $P$ is a first integral of the equation.

The general expression of iterative LODEs has been obtained in $[3,5]$ in explicit form in terms of the single arbitrary function $\mathfrak{q}$ upon which they depend. However, using a result of $[3,6]$, one can easily generate LODEs of maximal symmetry of a general order. For even $\operatorname{orders} n, 2 \leq n \leq 8$, the equations are as follows:

$$
\begin{aligned}
0= & \mathfrak{q} y+y_{x x}, \\
0= & 10 y_{x} \mathfrak{q}_{x}+10 \mathfrak{q} y_{x x}+3 y\left(3 \mathfrak{q}^{2}+\mathfrak{q}_{x x}\right)+y^{(4)} \\
0= & 7 y_{x x}\left(37 \mathfrak{q}^{2}+9 \mathfrak{q}_{x x}\right)+70 \mathfrak{q}_{x} y^{(3)}+y_{x}\left(518 \mathfrak{q} \mathfrak{q}_{x}\right. \\
& \left.+28 \mathfrak{q}^{(3)}\right)+35 \mathfrak{q} y^{(4)}+5 y\left(45 \mathfrak{q}^{3}+26 \mathfrak{q}_{x}^{2}+31 \mathfrak{q} \mathfrak{q}_{x x}\right. \\
& \left.+\mathfrak{q}^{(4)}\right)+y^{(6)} \\
0= & 168 y^{(3)}\left(47 \mathfrak{q} \mathfrak{q}_{x}+2 \mathfrak{q}^{(3)}\right)+42\left(47 \mathfrak{q}^{2}+9 \mathfrak{q}_{x x}\right) y^{(4)} \\
& +4 y_{x x}\left(3229 \mathfrak{q}^{3}+1773 \mathfrak{q} \mathfrak{q}_{x x}+45\left(33 \mathfrak{q}_{x}^{2}+\mathfrak{q}^{(4)}\right)\right) \\
& +6 y_{x}\left(6458 \mathfrak{q}^{2} \mathfrak{q}_{x}+524 \mathfrak{q} \mathfrak{q}^{(3)}\right. \\
& \left.+9\left(132 \mathfrak{q}_{x} \mathfrak{q}_{x x}+\mathfrak{q}^{(5)}\right)\right)+252 \mathfrak{q}_{x} y^{(5)}+84 \mathfrak{q} y^{(6)} \\
& +7 y\left(1575 \mathfrak{q}^{4}+1654 \mathfrak{q}^{2} \mathfrak{q}_{x x}+153 \mathfrak{q}_{2}^{2}+226 \mathfrak{q}_{x} \mathfrak{q}^{(3)}\right. \\
& \left.+8 \mathfrak{q}\left(347 \mathfrak{q}_{x}^{2}+10 \mathfrak{q}^{(4)}\right)+\mathfrak{q}^{(6)}\right)+y^{(8)}
\end{aligned}
$$

It can be easily verified that each of these equations is selfadjoint and thus admits a Lagrangian formulation in terms of the Euler-Lagrange operator. A Lagrangian $L_{n}$ for each of the equations $\Delta_{n}[y]=0$ of order $n$ in (13a)-(13d) can be taken in the form $L_{n}=y \Delta_{n}[y] / 2$, but this $n$th order Lagrangian can always be reduced to one of order $n / 2$.

As already noted, the results of $[3,6]$ can be used to generate iterative ODEs of any given order and thus we omit the listing of those iterative equations of odd orders $n=$ $3,5,7$ whose first integrals are also to be found. Although odd order equations are not known to possess a Lagrangian formulation, in order for a symmetry $\mathbf{v}$ of any such equation to give rise to a first integral it suffices that it be a divergence symmetry, that is, that it satisfies the divergence symmetry condition (11).

On the other hand, under the assumption that $u$ and $v$ are linearly independent solutions of (4), one can verify that for each even order $n \geq 4$ the most general divergence symmetry of each of (13b)-(13d) has the form

$$
\begin{aligned}
\mathbf{w} & \equiv \mathbf{w}^{n}=\mathbf{w}_{1}+\mathbf{w}_{2}, \\
\mathbf{w}_{1} & \equiv \mathbf{w}_{1}^{n}=\sum_{k=0}^{n-1} \lambda_{k} V_{k}, \quad \lambda_{k} \in \mathbb{R}, n=4,6,8, \\
\mathbf{w}_{2} & \equiv \mathbf{w}_{2}^{n}=\alpha F_{n}+\beta G_{n}+\gamma H_{n}, \quad \alpha, \beta, \gamma \in \mathbb{R} .
\end{aligned}
$$

In other words, for even orders $n \geq 4, W_{y}=y \partial_{y}$ is the only generator in (6a)-(6f) which is not a term of the divergence symmetry $\mathbf{w}$, and in particular $W_{y}$ itself is not a divergence symmetry. Similarly, for $n=2$, let $\mathbf{w}$ be an arbitrary divergence symmetry vector in the divergence symmetry algebra of (13a). Thus we have

$$
\mathbf{w}=\mathbf{w}_{1}+\mathbf{w}_{2}+\mathbf{w}_{3}+k_{3} W_{y},
$$

where $\mathbf{w}_{1}$ and $\mathbf{w}_{2}$ are like in (14a)-(14c) but with $n=2$ and

$$
\mathbf{w}_{3}=k_{1} C_{1}+k_{2} C_{2},
$$

where $k_{j}$ for $j=1,2,3$ are arbitrary scalars and $C_{1}$ and $C_{2}$ are the non-Cartan symmetries already introduced. Then in the divergence symmetry condition $\mathscr{D}(\mathbf{w})=0$ expressed as a polynomial in $y$ and its derivatives, it turns out that the coefficients of $y^{\prime \prime}$ and $y^{\prime} y^{\prime \prime}$ are $2 k_{3}$ and $-3\left(k_{1} u+k_{2} v\right)$, respectively. This shows that in the expression of $\mathbf{w}$ one must have $k_{j}=0$ for $j=1,2,3$. Consequently, $\mathbf{w}=\mathbf{w}_{1}+\mathbf{w}_{2}$ in this case also, and one readily verifies that any such $\mathbf{w}$ is indeed a divergence symmetry.

Let us now denote by $\mathscr{L}_{n}$ the Lie subalgebra of all divergence symmetries of $\mathfrak{g}_{n}$ for a given order $n$. We have thus established the following result.

Proposition 1. For every LODE of maximal symmetry of the most general form (13a)-(13d) and of even order $n$, a basis $\mathscr{B}_{n}$ of the Lie algebra $\mathscr{L}_{n}$ of its divergence symmetries is given by

$$
\mathscr{B}_{n}=\left\{F_{n}, G_{n}, H_{n}, V_{1}, \ldots, V_{n}\right\} .
$$

In particular, $\mathscr{L}_{n}$ has dimension $n+3$.

For equations of odd orders, the most general divergence symmetry $\mathbf{w}$ can be sought in the form

$$
\mathbf{w}=\mathbf{w}_{1}+\mathbf{w}_{2}+k_{1} W_{y}
$$

where $\mathbf{w}_{1}$ and $\mathbf{w}_{2}$ are like in (14a)-(14c) but with $n=3,5,7$ and $k_{1}$ is a scalar. Then in the divergence symmetry condition $\mathscr{D}(\mathbf{w})=0$ expressed as a polynomial in $y$ and its derivatives, the coefficient of $y^{(n+1)}$ is $2\left(\alpha u^{2}+2 \beta u v+\gamma v^{2}\right)$, and owing to the linear independence of $u$ and $v$ the vanishing of this coefficient implies that $\alpha=\beta=\gamma=0$. This reduces the expression of $\mathbf{w}$ to $\mathbf{w}=\mathbf{w}_{1}+k_{1} W_{y}$, and it is readily verified that the latter vector is always a divergence symmetry. We have thus established the following result. 
Proposition 2. For odd orders $n$, a basis $\mathscr{B}_{n}$ of $\mathscr{L}_{n}$ is given by

$$
\mathscr{B}_{n}=\left\{W_{y}, V_{1}, \ldots, V_{n}\right\} .
$$

In particular, $\mathscr{L}_{n}$ has dimension $n+1$.

It follows from (12) that, for each characteristic function $Q$, the corresponding first integral is given by

$$
P[y]=\int Q \Delta_{n}[y] d x .
$$

However, the evaluation of this integral is not straightforward. We shall derive this expression for each of the $n$ characteristics $Q=s_{k}(0 \leq k \leq n-1)$, where $s_{k}$ given in (3) are linearly independent solutions of the equation. The first integral associated with a given divergence symmetry generator $\mathbf{v}$ will be denoted by $\mathscr{F}(\mathbf{v})$ that associated with $V_{k} \equiv V_{n, k}$ by $\mathscr{F}_{k} \equiv \mathscr{F}_{n, k}$ for fixed $n$, and the first integral associated with the Lie algebra $\mathscr{A}_{n}$ generated by $V_{k}$ will be denoted by $\mathscr{F}^{n}$.

We consider explicitly the case $n=4$. In order to ensure that the equation reflects the fact that a given function $S$ is a solution, we solve $\Delta_{4}[S]=0$ for the coefficient $A_{0}$ of $y$ in (13b) and substitute the resulting expression back into $\mathrm{Q} \Delta_{4}[y]$. This yields

$$
\begin{aligned}
& Q \Delta_{4}[y]=S \Delta_{4}[y]=S\left[y^{(4)}+10\left(y_{x} \mathfrak{q}_{x}+\mathfrak{q} y_{x x}\right)\right. \\
& \left.\quad+A_{0} y\right] \\
& =S\left[y^{(4)}+10\left(y_{x} \mathfrak{q}_{x}+\mathfrak{q} y_{x x}\right)\right. \\
& \left.\quad+y \frac{-10 \mathfrak{q}_{x} S_{x}-10 a S_{x x}-S^{(4)}}{S}\right] \\
& =10 D\left(\mathfrak{q} S y_{x}-\mathfrak{q} y S_{x}\right)+D\left(S y^{(3)}-S_{x} y^{(2)}+S_{x x} y_{x}\right. \\
& \left.\quad-S^{(3)} y\right) .
\end{aligned}
$$

This shows that

$$
\begin{aligned}
P[y]= & 10\left(\mathfrak{q} S y_{x}-\mathfrak{q} S_{x} y\right)+S y^{(3)}-S_{x} y^{(2)}+S_{x x} y_{x} \\
& -S^{(3)} y+c
\end{aligned}
$$

for a certain constant of integration $c$ which will be omitted in the sequel. Letting $S=s_{k}$ as in (3), it follows that the most general expression of first integrals generated by solutions of $\Delta_{4}[y]=0$ has the form

$$
\begin{aligned}
\mathscr{F}^{4}= & \sum_{k=0}^{3} \alpha_{k} \mathscr{F}_{4, k}, \quad \alpha_{k} \in \mathbb{R}, \\
\mathscr{F}_{4, k}= & 10\left(\mathfrak{q} S y_{x}-\mathfrak{q} S_{x} y\right)+S y^{(3)}-S_{x} y^{(2)}+S_{x x} y_{x} \\
& -S^{(3)} y, \\
S= & s_{k}=u^{3-k} v^{k}, \quad(0 \leq k \leq 3) .
\end{aligned}
$$

By proceeding in the same manner as for $n=4$, using identities such as

$$
\begin{aligned}
& D\left(f S y_{x}-f y S_{x}\right)=S D\left(f y_{x}\right)-y D\left(f S_{x}\right) \\
& S y^{(m)}+(-1)^{m-1} y S^{(m)} \\
& \quad=D\left[\sum_{j=0}^{m-1}(-1)^{j} S^{(j)} y^{(m-1-j)}\right]
\end{aligned}
$$

where $f=f(x)$ is any given function and $m \in \mathbb{N}=$ $\{1,2,3, \ldots\}$, one obtains the first integrals for all other equations of orders $n \leq 8$. We first list the expressions of first integrals found starting with even order equations. Thus for equations of order $n=2$ one has

$$
\begin{aligned}
\mathscr{F}^{2} & =\sum_{k=0}^{1} \alpha_{k} \mathscr{F}_{2, k}, \quad \alpha_{k} \in \mathbb{R}, \\
\mathscr{F}_{2, k} & =S y^{\prime}-y S^{\prime}, \\
S & =s_{k}=u^{1-k} v^{k}, \quad(0 \leq k \leq 1) .
\end{aligned}
$$

For the order $n=6$ one has

$$
\begin{aligned}
& \mathscr{F}^{6}=\sum_{k=0}^{5} \alpha_{k} \mathscr{F}_{6, k}, \quad \alpha_{k} \in \mathbb{R}, \\
& \mathscr{F}_{6, k}=S y^{(5)}+y_{x x}\left(35 S \mathfrak{q}_{x}-35 \mathfrak{q} S_{x}-S^{(3)}\right)+(35 \mathfrak{q} S \\
& \left.\quad+S_{x x}\right) y^{(3)}+y_{x}\left(259 \mathfrak{q}^{2} S+28 S \mathfrak{q}_{x x}+35 \mathfrak{q} S_{x x}\right. \\
& \left.\quad+S^{(4)}\right)-S_{x} y^{(4)}+y\left(-259 \mathfrak{q}^{2} S_{x}-28 S_{x} \mathfrak{q}_{x x}\right. \\
& \left.\quad-35 \mathfrak{q}_{x} S_{x x}-35 \mathfrak{q} S^{(3)}-S^{(5)}\right), \\
& S=s_{k}=u^{5-k} v^{k}, \quad(0 \leq k \leq 5) .
\end{aligned}
$$

On the other hand, for $n=8$, one has

$$
\begin{aligned}
\mathscr{F}^{8} & =\sum_{k=0}^{7} \alpha_{k} \mathscr{F}_{8, k}, \quad \alpha_{k} \in \mathbb{R}, \\
\mathscr{F}_{8, k} & =y^{(3)}\left(1974 \mathfrak{q}^{2} S-84 \mathfrak{q}_{x} S_{x}+210 S \mathfrak{q}_{x x}+84 \mathfrak{q} S_{x x}\right. \\
& \left.+S^{(4)}\right)+y_{x x}\left(-1974 \mathfrak{q}^{2} S_{x}-126 S_{x} \mathfrak{q}_{x x}+126 S \mathfrak{q}^{(3)}\right. \\
& \left.+84 \mathfrak{q}\left(47 S \mathfrak{q}_{x}-S^{(3)}\right)-S^{(5)}\right)+\left(168 S \mathfrak{q}_{x}-84 \mathfrak{q} S_{x}\right. \\
& \left.-S^{(3)}\right) y^{(4)}+\left(84 \mathfrak{q} S+S_{x x}\right) y^{(5)}+y_{x}\left(12916 \mathfrak{q}^{3} S\right. \\
& +1974 \mathfrak{q}^{2} S_{x x}+126 \mathfrak{q}_{x x} S_{x x}+84 \mathfrak{q}_{x} S^{(3)}
\end{aligned}
$$




$$
\begin{aligned}
& +6 S\left(332 \mathfrak{q}_{x}^{2}+9 \mathfrak{q}^{(4)}\right)+12 \mathfrak{q}\left(262 S \mathfrak{q}_{x x}+7 S^{(4)}\right) \\
& \left.+S^{(6)}\right)-S_{x} y^{(6)}+y\left(-12916 \mathfrak{q}^{3} S_{x}-1992 \mathfrak{q}_{x}^{2} S_{x}\right. \\
& -3144 \mathfrak{q} S_{x} \mathfrak{q}_{x x}-3948 \mathfrak{q} \mathfrak{q}_{x} S_{x x}-126 S_{x x} \mathfrak{q}^{(3)} \\
& -1974 \mathfrak{q}^{2} S^{(3)}-210 \mathfrak{q}_{x x} S^{(3)}-54 S_{x} \mathfrak{q}^{(4)}-168 \mathfrak{q}_{x} S^{(4)} \\
& \left.-84 \mathfrak{q} S^{(5)}-S^{(7)}\right)+S y^{(7)} \\
S= & s_{k}=u^{7-k} v^{k}, \quad(0 \leq k \leq 7)
\end{aligned}
$$

The algorithm for finding first integrals for iterative equations of odd order $n$ is different from that for even order equations described above. One can always write an iterative equation $\Delta_{n}[y]=0$ of the form (2) in the form $y^{(n)}+F_{n}\left(x, y^{(n-2)}\right)=$ 0 . Therefore, the characteristic form of the conservation law associated with a solution $S=s_{k}$ takes the form

$$
\begin{aligned}
S \Delta_{n}[y]= & S \Delta_{n}[y]+S^{(n)} y+y F_{n}\left(x, S^{(m)}\right) \\
= & {\left[S y^{(n)}+S^{(n)} y\right] } \\
& +\left[S F_{n}\left(x, y^{(n-2)}\right)+y F_{n}\left(x, S^{(n-2)}\right)\right] .
\end{aligned}
$$

Then, using the identities in (24a) and (24b), one naturally integrates each of the two expressions in square brackets appearing in (28b) to obtain the first integrals. The resulting expressions are given as follows for the order $n=3$.

$$
\begin{aligned}
\mathscr{F}^{3} & =\sum_{k=0}^{2} \alpha_{k} \mathscr{F}_{3, k}, \quad \alpha_{k} \in \mathbb{R}, \\
\mathscr{F}_{3, k} & =4 \mathfrak{q} S y-S_{x} y_{x}+y S_{x x}+S y_{x x}, \\
S & =s_{k}=u^{2-k} v^{k}, \quad(0 \leq k \leq 2) .
\end{aligned}
$$

For $n=5$, one has

$$
\begin{aligned}
\mathscr{F}^{5}= & \sum_{k=0}^{4} \alpha_{k} \mathscr{F}_{5, k}, \quad \alpha_{k} \in \mathbb{R}, \\
\mathscr{F}_{5, k} & \\
= & \left(20 \mathfrak{q} S+S_{x x}\right) y_{x x}+y_{x}\left(10 S \mathfrak{q}_{x}-20 \mathfrak{q} S_{x}-S^{(3)}\right) \\
& +y\left(64 \mathfrak{q}^{2} S+10 \mathfrak{q}_{x} S_{x}+8 S \mathfrak{q}_{x x}+20 \mathfrak{q} S_{x x}+S^{(4)}\right) \\
& +S y^{(4)}-S_{x} y^{(3)}, \\
S= & s_{k}=u^{4-k v^{k}, \quad(0 \leq k \leq 4) .}
\end{aligned}
$$

Finally, for $n=7$, the first integrals are given by

$$
\begin{aligned}
\mathscr{F}^{7} & =\sum_{k=0}^{6} \alpha_{k} \mathscr{F}_{7, k}, \quad \alpha_{k} \in \mathbb{R}, \\
\mathscr{F}_{7, k} & =\left(84 S \mathfrak{q}_{x}-56 \mathfrak{q} S_{x}-S^{(3)}\right) y^{(3)}+y_{x x}\left(784 \mathfrak{q}^{2} S\right. \\
& \left.-28 \mathfrak{q}_{x} S_{x}+84 S \mathfrak{q}_{x x}+56 \mathfrak{q} S_{x x}+S^{(4)}\right)+(56 \mathfrak{q} S \\
& \left.+S_{x x}\right) y^{(4)}+y_{x}\left(784 \mathfrak{q} S \mathfrak{q}_{x}-784 \mathfrak{q}^{2} S_{x}-56 S_{x} \mathfrak{q}_{x x}\right. \\
& \left.-28 \mathfrak{q}_{x} S_{x x}+28 S \mathfrak{q}^{(3)}-56 \mathfrak{q} S^{(3)}-S^{(5)}\right)-S_{x} y^{(5)} \\
& +y\left(2304 \mathfrak{q}^{3} S+784 \mathfrak{q}^{2} S_{x x}+84 \mathfrak{q}_{x x} S_{x x}+28 S_{x} \mathfrak{q}^{(3)}\right. \\
& +84 \mathfrak{q}_{x} S^{(3)}+12 S\left(33 \mathfrak{q}_{x}^{2}+\mathfrak{q}^{(4)}\right) \\
& \left.+8 \mathfrak{q}\left(98 \mathfrak{q}_{x} S_{x}+78 S \mathfrak{q}_{x x}+7 S^{(4)}\right)+S^{(6)}\right)+S y^{(6)}, \\
S= & s_{k}=u^{6-k} v^{k}, \quad(0 \leq k \leq 6) .
\end{aligned}
$$

The results obtained thus far in this section about first integrals can be summarized as follows for all orders $n$ such that $2 \leq n \leq 8$.

Theorem 3. (a) For even $n$, the first integrals $\mathscr{F}_{n, k}$ associated with the solution symmetries $V_{k}=s_{k} \partial_{y}$ are given by (23b) and (25)-(27), while they are given for odd $n$ by (29)-(31).

(b) For each order $n$, the $n$ first integrals $\mathscr{F}_{n, k}(0 \leq k \leq$ $n-1)$ are linearly independent.

(c) If for each $n$ and $k$ we view $\mathscr{F}_{n, k}=\mathscr{F}_{n, k}[y, q]$ as a differential function of $y$ and $\mathfrak{q}$, then $\mathscr{F}_{n, k}$ is linear in $y$ (and its derivatives) and polynomial in $\mathfrak{q}$.

First integrals are often required to be in a form in which they depend only on the parameters of the underlying differential equation and are not in particular expressed in terms of a particular solution of the equation. The first integrals we have found can indeed be expressed solely in terms of the dependent variable $y$ and the coefficient $\mathfrak{q}$ and their derivatives. For instance, in the case of equations of odd order $n$, given that $\mathscr{F}_{n, k}$ are symmetric functions of $S$ and $y$ and are required to be constant only on the solution space, the required type of first integrals can be obtained simply by letting $S=y$ in the expression of $\mathscr{F}_{n, k}$. Denoting by $\mathscr{F}_{n}^{y}$ the resulting first integrals for each odd order $n$ of the equation yields the following expressions:

$$
\begin{aligned}
\mathscr{F}_{3}^{y}= & 4 \mathfrak{q} y^{2}-y_{x}^{2}+2 y y_{x x}, \\
\mathscr{F}_{5}^{y}= & 20 y \mathfrak{q}_{x} y_{x}-20 \mathfrak{q} y_{x}^{2}+8 y^{2}\left(8 \mathfrak{q}^{2}+\mathfrak{q}_{x x}\right) \\
& +40 \mathfrak{q} y y_{x x}+y_{x x}^{2}-2 y_{x} y^{(3)}+2 y y^{(4)},
\end{aligned}
$$




$$
\begin{aligned}
\mathscr{F}_{7}^{y}= & -56 y_{x}^{2}\left(14 \mathfrak{q}^{2}+\mathfrak{q}_{x x}\right)-56 \mathfrak{q}_{x} y_{x} y_{x x} \\
& +56 y\left(28 \mathfrak{q}^{2}+3 \mathfrak{q}_{x x}\right) y_{x x}+56 \mathfrak{q} y_{x x}^{2} \\
& +56 y y_{x}\left(28 \mathfrak{q} \mathfrak{q}_{x}+\mathfrak{q}^{(3)}\right)+168 y \mathfrak{q}_{x} y^{(3)} \\
& -112 \mathfrak{q} y_{x} y^{(3)}-y^{(3)^{2}} \\
& +12 y^{2}\left(192 \mathfrak{q}^{3}+33 \mathfrak{q}_{x}^{2}+52 \mathfrak{q} \mathfrak{q}_{x x}+\mathfrak{q}^{(4)}\right) \\
& +112 \mathfrak{q} y y^{(4)}+2 y_{x x} y^{(4)}-2 y_{x} y^{(5)}+2 y y^{(6)}
\end{aligned}
$$

We notice that, for each $n$, the first integral $\mathscr{F}_{n}^{y}=\mathscr{F}_{n}^{y}[y, \mathfrak{q}]$ viewed as a differential function of $y$ and $\mathfrak{q}$ is quadratic homogeneous in $y$ and polynomial in $\mathfrak{q}$.

In [18], first integrals of the trivial equation $y^{(n)}=0$ were explicitly computed for $n=2,3, \ldots, 6$, and they are directly expressed in terms of the linearly independent ones thus obtained. The method used in that paper for finding the first integrals $F$ associated with each symmetry $\mathbf{v}$ of the equation is direct computation done by solving the boundary value problem

$$
\begin{aligned}
& \mathbf{v}^{[n-1]}(F)=0, \\
& \left.\frac{d F}{d x}\right|_{y^{(n)}=0}=0 .
\end{aligned}
$$

However, the calculations done in [18] are only a special case of those done in this paper for equations of maximal symmetry and low order $n \leq 8$. Indeed, we have considered equations of maximal symmetry in their most general form (13a)-(13d) and not in the simpler canonical form $y^{(n)}=$ 0 . Of course the first integrals we have found here from a variational principle all satisfy (33a) and (33b).

It follows from Liouville's formula that if $s_{1}, \ldots, s_{n}$ are linearly independent solutions of an equation of the form (2), then $n$ linearly independent first integrals of the equations are given by the Wronskians

$$
\begin{aligned}
R_{j}[y]=w\left(s_{1}, \ldots, s_{j-1}, y, s_{j+1}, \ldots, s_{n}\right), & \\
& j=1, \ldots, n .
\end{aligned}
$$

However, this direct calculation does not relate the first integral to any variational symmetry of the equation and thus cannot exploit the various properties of such symmetries. Moreover, it requires the knowledge of the complete set of linear independent solutions of the equation itself, while those we found involve only two linearly independent solutions of the second-order source equation. In addition, these two symbolic solutions are not required to be known explicitly but are only assumed to satisfy the second-order equation. In fact, we have ultimately expressed the first integrals found solely in terms of $\mathfrak{q}, y$ and their derivatives.

\section{Hamiltonian Formulation}

In order to gain more insight into the properties of LODEs of maximal symmetry, we shall move one step forward in this section by finding a Hamiltonian formulation of some of these equations and investigate some of its properties. Indeed, in the case of scalar equations, the study in the previous sections was largely facilitated thanks to formula (3) which provides the general solution of any iterative LODE through a very simple superposition principle in terms of two linearly independent solutions $u$ and $v$ of the second-order source equation (4). Thus one of our ultimate goals in this undertaking is to obtain an extension of such a formula to Hamiltonian systems associated with equations of maximal symmetry.

For second-order equations, the transformation into a Hamiltonian system is usually achieved by the common method via the Legendre transformation by defining the conjugate momenta as $p=\partial L / \partial y$, where $L$ is the Lagrangian. However, for higher-order equations, one can make use of the Jacobi-Ostrogradski generalized momenta [19, 20].

Indeed, consider in this more general approach a given $n$th order Euler-Lagrange equation $E(L)=0$, where $E$ is the usual Euler-Lagrange operator (see [7, Page 250]) applied to a Lagrangian $L[y]=L\left(x, y^{(m)}\right)$. In the latter equality, $m$ can be a multi-index of order $n / 2$ and $y$ a multivariable but which in our own considerations will be limited to a single dependent variable of the independent variable $x$. Then the generalized momenta are defined by the expressions

$$
\begin{aligned}
p_{1} & =\frac{\partial L}{\partial y_{x}}-D_{x}\left(\frac{\partial L}{\partial y_{x x}}\right)+\cdots \\
& +(-1)^{m-1} D_{x}^{m-1}\left(\frac{\partial L}{\partial y^{(m)}}\right) \\
p_{2} & =\frac{\partial L}{\partial y_{x x}}-D_{x}\left(\frac{\partial L}{\partial y_{x x x}}\right)+\cdots \\
& +(-1)^{m-2} D_{x}^{m-2}\left(\frac{\partial L}{\partial y^{(m)}}\right) \\
\vdots & \\
p_{m} & =\left(\frac{\partial L}{\partial y^{(m)}}\right) .
\end{aligned}
$$

As for the canonical coordinates, they are given by

$$
q_{j}=y^{(j-1)}, \quad \text { for } j=1, \ldots, m
$$

The corresponding Hamiltonian function then takes the form

$$
H=p_{1} q_{2}+p_{2} q_{3}+\cdots+p_{m-1} q_{m}+p_{m} y^{(m)}-L,
$$


and in the new coordinates $\left(x,\left(q_{j}\right),\left(p_{j}\right)\right)$ the HamiltonCartan equations are given as usual by

$$
\begin{aligned}
& \frac{d q_{j}}{d x}=\frac{\partial H}{\partial p_{j}}, \\
& \frac{d p_{j}}{d x}=-\frac{\partial H}{\partial q_{j}} .
\end{aligned}
$$

For each LODE $\Delta_{n}[y]=0$, it is well known [7] as already noted that when the equation is of the Euler-Lagrange type, a Lagrangian $L$ is found by setting

$$
L=\frac{1}{2} y \Delta_{n}[y] .
$$

Moreover, this $n$th order Lagrangian can always be reduced to one of order $n / 2$ by eliminating some null Lagrangian terms from it. Denoting as usual by $L_{n}$ the reduced Lagrangian corresponding $n$ listed in (13a)-(13d), it is found that

$$
\begin{aligned}
& L_{2}=\frac{1}{2}\left(\mathfrak{q} y^{2}-y_{x}^{2}\right) \\
& L_{4}=-5 \mathfrak{q} y_{x}^{2}+\frac{3}{2} y^{2}\left(3 \mathfrak{q}^{2}+\mathfrak{q}_{x x}\right)+\frac{1}{2} y_{x x}^{2},
\end{aligned}
$$

for the first two equations. In case $n=2$, the canonical variables are $(q, p)$ with $q=y$ and $p=\partial L / \partial y_{x}=-y_{x}$, and, by the Legendre transformation, the Hamiltonian in this case has the expression

$$
H_{2}=-\left(y_{x} p-L\right)=-\frac{1}{2}\left(p^{2}+\mathfrak{q} q^{2}\right)
$$

with corresponding Hamiltonian system

$$
\begin{aligned}
& \dot{q}=-p, \\
& \dot{p}=\mathfrak{q} q,
\end{aligned}
$$

where we have $\dot{F}=d F / d x$, for any function $F=F(x)$. It turns out that (42) is linear as was to be expected given that $\mathrm{H}_{2}$ is quadratic, but also (42) coincides with the standard representation of the second-order source equation (4) as a first-order system. Thus two linearly independent solutions of $(42)$ are $\left(u,-u_{x}\right)$ and $\left(v,-v_{x}\right)$, with the usual notations.

For $n=4$, the canonical coordinates $\left(q_{1}, q_{2}, p_{1}, p_{2}\right)$ are obtained more explicitly from (35) and (36) as

$$
\begin{aligned}
& q_{1}=y \\
& q_{2}=y_{x} \\
& p_{1}=\frac{\partial L_{4}}{\partial y_{x}}-\frac{d}{d x}\left(\frac{\partial L_{4}}{y_{x x}}\right)=-10 \mathfrak{q} y_{x}-y^{(3)}, \\
& p_{2}=\frac{\partial L_{4}}{\partial y_{x x}}=y_{x x}
\end{aligned}
$$

and the quadratic Hamiltonian $H_{4}$ has the expression

$$
\begin{aligned}
H_{4} & =p_{1} y_{x}+p_{2} y_{x x}-L_{4} \\
& =\frac{1}{2}\left(p_{2}^{2}-9 \mathfrak{q}^{2} q_{1}^{2}+2 p_{1} q_{2}+10 \mathfrak{q} q_{2}^{2}-3 q_{1}^{2} \ddot{\mathfrak{q}}\right),
\end{aligned}
$$

whereas the corresponding linear Hamiltonian system takes the form

$$
\begin{aligned}
& \dot{q}_{1}=q_{2}, \\
& \dot{q}_{2}=p_{2}, \\
& \dot{p}_{1}=3 q_{1} \ddot{\mathfrak{q}}+9 \mathfrak{q}^{2} q_{1}, \\
& \dot{p}_{2}=-p_{1}-10 \mathfrak{q} q_{2} .
\end{aligned}
$$

A question which is worth considering at this point is whether solutions to the Hamiltonian system (45) can also be obtained through a simple superposition formula from the solutions of the second-order system (42), as in the case of scalar equations of maximal symmetry. By construction of (45), this question is already positively answered by formulas (43) which provide an explicit expression for the unknown variables $q_{j}$ and $p_{j}$ directly in terms of the solutions of the scalar source equation (4). Indeed, if $s=s(x)$ is any given function of $x$, then (43) shows that $S=\left\{s, s_{x},-10 \mathfrak{q} s_{x}-\right.$ $\left.s^{(3)}, s_{x x}\right\}$ is a solution to (45) if and only if $s$ is a solution to the corresponding fourth-order scalar equation (13b). Therefore, a complete set of linearly independent solutions for (45) is given by

$$
\begin{aligned}
& S_{k}=\left\{s_{k},\left(s_{k}\right)_{x},-10 \mathfrak{q}\left(s_{k}\right)_{x}-s_{k}^{(3)},\left(s_{k}\right)_{x x}\right\}, \\
& s_{k}=u^{3-k} v^{k}, \quad k=0, \ldots, 3,
\end{aligned}
$$

where as usual $u$ and $v$ are two linearly independent solutions of (4), using also the fact that $\mathfrak{q}=w\left(u_{x}, v_{x}\right)$ by (5). Note that, for any choice of $s$ in the expression of $S$, all equations in (45) are always satisfied, except for the third one which reduces exactly to (13b).

More generally, it follows from (35) and (36) that the general solution to every Hamiltonian system derived from an equation of maximal symmetry as those given in (13a)-(13d) can be found by a mere superposition formula from the solutions of the second-order source equation (4). It will therefore be useful to obtain a characterization of the equivalence class under point transformations of these nontrivial families of Hamiltonian systems depending on the arbitrary function $\mathfrak{q}$, as well as its symmetry properties.

Restricting our attention for now to the fourth-order system (45), the existence of the superposition formula (46) indicates that this system of equations can be reduced in order, and we know this is possible in particular if the system has a two-parameter abelian symmetry subgroup. As is well known for Hamiltonian systems, symmetries are essentially in one-one correspondence with first integrals $F$ of the system, the determining equation of which is given by

$$
\begin{aligned}
& \frac{\partial F}{\partial x}+\{F, H\}=0 \Longleftrightarrow \\
& \frac{\partial F}{\partial x}+\widehat{\mathbf{v}}_{H}(F)=0 .
\end{aligned}
$$

In (47a) and (47b), $\{$,$\} is the associated Poisson bracket for$ the canonical coordinates and $\widehat{\mathbf{v}}_{H}$ is the Hamiltonian vector 
field associated with the Hamiltonian function $H$. In the case of (45), we have

$$
\begin{aligned}
\widehat{\mathbf{v}}_{H}= & -q_{2} \partial_{q_{1}}-p_{2} \partial_{q_{2}}-3 q_{1}\left(3 \mathfrak{q}^{2}+\ddot{\mathfrak{q}}\right) \partial_{p_{1}} \\
& +\left(p_{1}+10 \mathfrak{q} q_{2}\right) \partial_{p_{2}} .
\end{aligned}
$$

Due to the arbitrariness of the function $\mathfrak{q}=\mathfrak{q}(x)$ in the expression of $\widehat{\mathbf{v}}_{H}$, it does not seem to be possible to solve directly the system corresponding to (48). However, it should be possible to find these first integrals as in the scalar case by exploiting the fact that $u$ and $v$ are solutions of (4). Here, we look at what happens when $\mathfrak{q}=0$. In this case, we find the four functionally independent first integrals

$$
\begin{aligned}
& f_{1}=\frac{1}{6}\left(-x^{3} p_{1}+3 x^{2} p_{2}+6 q_{1}+6 x q_{2}\right), \\
& f_{2}=\frac{1}{2}\left(-x^{2} p_{1}+2 x p_{2}+2 q_{2}\right), \\
& f_{3}=-x p_{1}+p_{2}, \\
& f_{4}=p_{1} .
\end{aligned}
$$

We clearly have, for instance, $\left\{f_{1}, f_{2}\right\}=0$ and thus $\widehat{\mathbf{v}}_{f_{1}}$ and $\widehat{\mathbf{v}}_{f_{2}}$ generate an abelian Hamiltonian symmetry group of order two of the equation. Therefore, system (45) can be reduced in order by four and hence its general solution can be found by a mere superposition formula from those of a secondorder reduced system, that is, in particular from the solutions of the source equation (4). Moreover, the corresponding system with two degrees of freedom (45) is maximally superintegrable in the sense of $[21,22]$. It is our belief that all Hamiltonian systems derived from equations of maximal symmetry (13a)-(13d) should satisfy similar properties in their most general form. But this investigation is postponed to a future research project.

\section{Concluding Remarks}

We would like to comment on two other possible methods to find the first integrals obtained in this paper and more generally the first integrals of any system of differential equations. The first method is due to two important results recently obtained in [6]. The first of these two results is an efficient algorithm for generating equations of maximal symmetry of arbitrary orders and of the most general form solely in terms of the coefficient $\mathfrak{q}$ and its derivatives. The second result is the point transformation

$$
\begin{aligned}
& \zeta=\frac{v}{u}, \\
& \theta=k_{0} u^{1-n} y,
\end{aligned}
$$

where $k_{0}$ is an arbitrary constant, which maps the canonical form $\theta^{(n)}=0$ to the most general form $\Delta_{n}[y]=\Delta_{n}[y, \mathfrak{q}]$ of a LODE of maximal symmetry. Thanks to these two results, to find first integrals of linear equations of maximal symmetry in their most general form, it suffices to find that for the canonical form of the corresponding equation. Indeed, the first integral of the transformed equation under (50) is just the transformed version under (50) of the first integral of the original equation. For instance, the most general first integral $\mathscr{F}$ associated with solution symmetries of $\theta^{(2)}=0$ is easily found to be given by $\mathscr{F}=-a_{1} \theta+\left(a_{0}+a_{1} \zeta\right) \theta^{\prime}$, for some arbitrary constants $a_{0}$ and $a_{1}$. Under (50), $\mathscr{F}$ is simply transformed into (25). A research project is currently in progress to use this method to find first integrals for the most general forms of linear and nonlinear ODEs of maximal symmetry and of a general order.

Most often, to find first integrals of an $n$th order differential equation $\Delta_{n}[y]=0$, one looks for an integrating factor $\Pi[y]$ as a differential function of $y$ of order at most $n-1$ such that the expression $\Pi[y] \Delta_{n}[y]$ is a null Lagrangian, that is, such that $E\left(\Pi[y] \Delta_{n}[y]\right)=0$, where $E$ is the Euler operator. Indeed, the latter equality holds if and only if $\Pi[y] \Delta_{n}[y]=\operatorname{div} P$ is a total divergence for a certain differential function $P=P[y]$. Thus, in the case of ODEs, when $\Pi[y]$ is a finite expression, $P[y]$ is a first integral and may be expressed as the integral of $\Pi[y] \Delta_{n}[y]$. The first problem with this method is to find the integrating factor $\Pi[y]$. This can be achieved by an application of Nother's theorem as we did in this paper. There are several other more or less similar methods based on adjoint symmetries or other types of symmetries [23-25] or on more direct methods $[26,27]$ achieved by solving equations similar to $E\left(\Pi[y] \Delta_{n}[y]\right)=0$. The second problem with these rather common methods is that once an integrating factor has been found the components of the vector $P[y]$ are obtained as integrals of generally complicated expressions, and thus evaluating such integrals remains usually challenging as it was the case in this paper.

\section{Competing Interests}

The author declares that there are no competing interests regarding the publication of this article.

\section{References}

[1] J. Krause and L. Michel, "Equations différentielles linéaires d'ordre $\mathrm{n}>2$ ayant une algèbre de Lie de symétrie de dimension $\mathrm{n}+4$," Comptes Rendus de l'Académie des Sciences. Paris, vol. 307, Article ID 905910, pp. 905-910, 1988.

[2] S. Lie, "Classification und Integration von gewöhnlichen Differentialgleichungen zwischenxy, die eine Gruppe von Transformationen gestatten," Mathematische Annalen, vol. 32, no. 2, pp. 213-281, 1888.

[3] J.-C. Ndogmo and F. M. Mahomed, "On certain properties of linear iterative equations," Central European Journal of Mathematics, vol. 12, no. 4, pp. 648-657, 2014.

[4] J. C. Ndogmo, "Coefficient characterization of linear differential equations with maximal symmetries," Communications in Nonlinear Science and Numerical Simulation, vol. 19, no. 5, pp. 1270-1278, 2014.

[5] F. M. Mahomed and P. G. Leach, "Symmetry Lie algebras of $n$th order ordinary differential equations," Journal of Mathematical Analysis and Applications, vol. 151, no. 1, pp. 80-107, 1990. 
[6] J. C. Ndogmo, "Generation and identification of ordinary differential equations of maximal symmetry algebra," Abstract and Applied Analysis, vol. 2016, Article ID 1796316, 9 pages, 2016.

[7] P. J. Olver, Applications of Lie groups to Differential Equations, vol. 107 of Graduate Texts in Mathematics, Springer-Verlag, New York, NY, USA, 1986.

[8] E. Noether, "Invariante variationsprobleme," Math-Phys Kl Heft, pp. 235-257, 1918.

[9] C. Nitsch, "Some stability properties involving first integrals," Rendiconti dell'Accademia delle Scienze Fisiche e Matemàtiche di Napoli, vol. 72, pp. 29-40, 2005.

[10] Z. Rzesztko, "Strong stability of linear symplectic actions and the orbit method," Buletinul Academiei de Stiinte a Republicii Moldova. Matematica, no. 2, pp. 99-103, 2005.

[11] M. F. Singer, "Liouvillian first integrals of differential equations," Transactions of the American Mathematical Society, vol. 333, no. 2, pp. 673-688, 1992.

[12] J. Chavarriga, H. Giacomini, J. Giné, and J. Llibre, "Darboux integrability and the inverse integrating factor," Journal of Differential Equations, vol. 194, no. 1, pp. 116-139, 2003.

[13] J. Giné and M. Grau, "Weierstrass integrability of differential equations," Applied Mathematics Letters, vol. 23, no. 5, pp. 523526, 2010.

[14] J. Giné, "Reduction of integrable planar polynomial differential systems," Applied Mathematics Letters. An International Journal of Rapid Publication, vol. 25, no. 11, pp. 1862-1865, 2012.

[15] A. R. Ünal, "Exact solutions of the Zakharov equations by using the first integral method," Communications de la Faculté des Sciences de l'Université d'Ankara. Séries A1. Mathematics and Statistics, vol. 61, pp. 9-16, 2012.

[16] S. Lie, Vorlesungüber Continuerliche Gruppen Mit Geometrischen und Anderen Anwendungen Bearbeitet und Herausgegeben von Dr. G. Scheffers, Teubner, Liepzig, Germany, 1893.

[17] C. Muriel, J. L. Romero, and P. J. Olver, "Variational $C^{\infty}$ symmetries and Euler-Lagrange equation," Journal of Differential Equations, vol. 222, no. 1, pp. 164-184, 2006.

[18] G. P. Flessas, K. S. Govinder, and P. G. L. Leach, "Characterisation of the algebraic properties of first integrals of scalar ordinary differential equations of maximal symmetry," Journal of Mathematical Analysis and Applications, vol. 212, no. 2, pp. 349-374, 1997.

[19] P. Guha, A. Ghose Choudhury, and A. S. Fokas, "Hamiltonians and conjugate Hamiltonians of some fourth-order nonlinear ODEs," Nonlinear Analysis: Theory, Methods \& Applications, vol. 75, no. 4, pp. 2126-2138, 2012.

[20] M. C. Nucci and P. G. Leach, "The method of Ostrogradsky, quantization, and a move toward a ghost-free future," Journal of Mathematical Physics, vol. 50, no. 11, 2009.

[21] P. Winternitz and I. Yurdusen, "Integrable and superintegrable systems with spin in three-dimensional Euclidean space," Journal of Physics A, vol. 42, no. 38, Article ID 385203, 20 pages, 2009.

[22] R. G. Smirnov and P. Winternitz, "A class of superintegrable systems of Calogero type," Journal of Mathematical Physics, vol. 47, no. 9, Article ID 093505, 8 pages, 2006.

[23] S. C. Anco and G. Bluman, "Integrating factors and first integrals for ordinary differential equations," European Journal of Applied Mathematics, vol. 9, no. 3, pp. 245-259, 1998.

[24] S. V. Meleshko, S. Moyo, C. Muriel, J. L. Romero, P. Guha, and A. G. Choudhury, "On first integrals of second-order ordinary differential equations," Journal of Engineering Mathematics, vol. 82, pp. 17-30, 2013.
[25] R. Naz, F. M. Mahomed, and D. P. Mason, "Comparison of different approaches to conservation laws for some partial differential equations in fluid mechanics," Applied Mathematics and Computation, vol. 205, no. 1, pp. 212-230, 2008.

[26] S. C. Anco and G. Bluman, "Direct construction method for conservation laws of partial differential equations. Part II: general treatment," European Journal of Applied Mathematics, vol. 13, no. 5, pp. 567-585, 2002.

[27] T. Wolf, "A comparison of four approaches to the calculation of conservation laws," European Journal of Applied Mathematics, vol. 13, no. 2, pp. 129-152, 2002. 


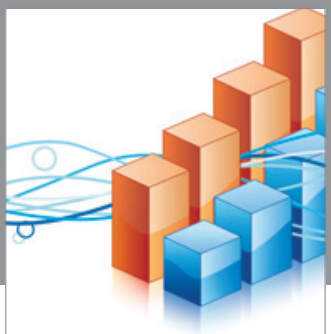

Advances in

Operations Research

vatem alat4

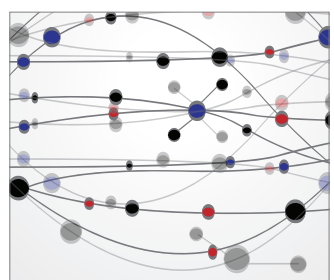

\section{The Scientific} World Journal
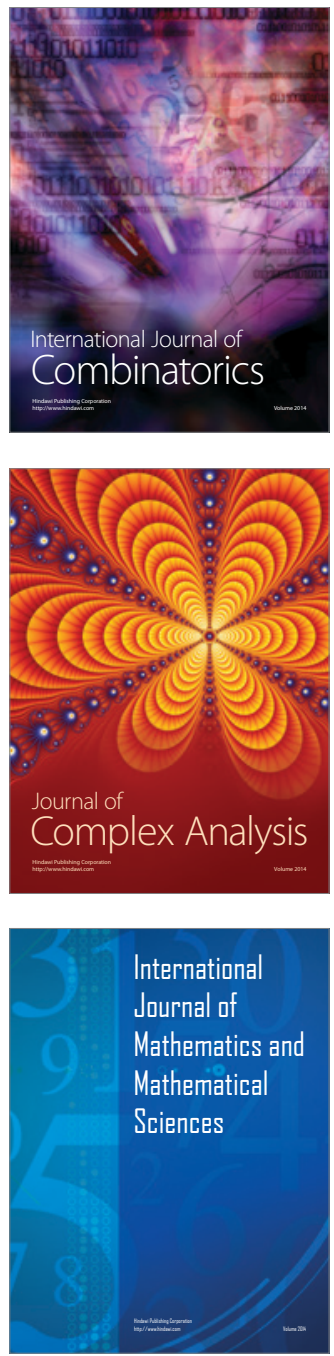
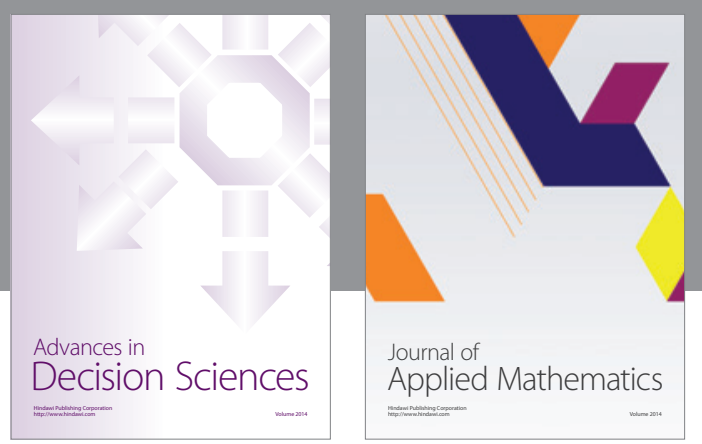

Algebra

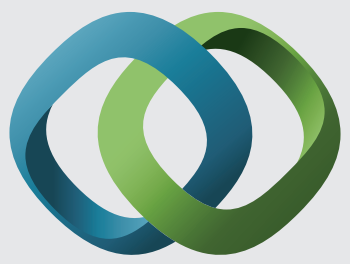

\section{Hindawi}

Submit your manuscripts at

https://www.hindawi.com
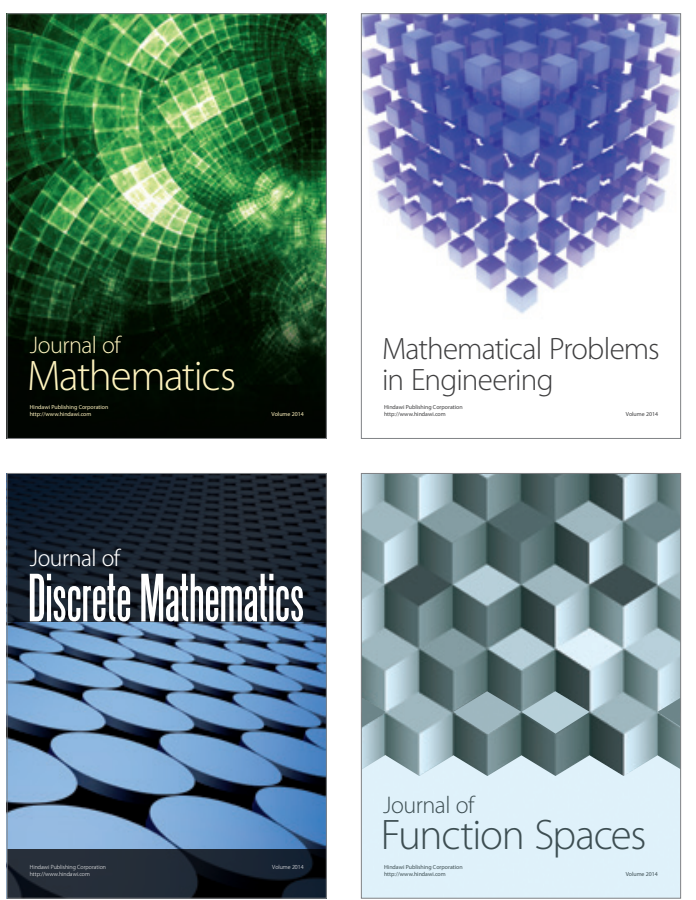

Mathematical Problems in Engineering
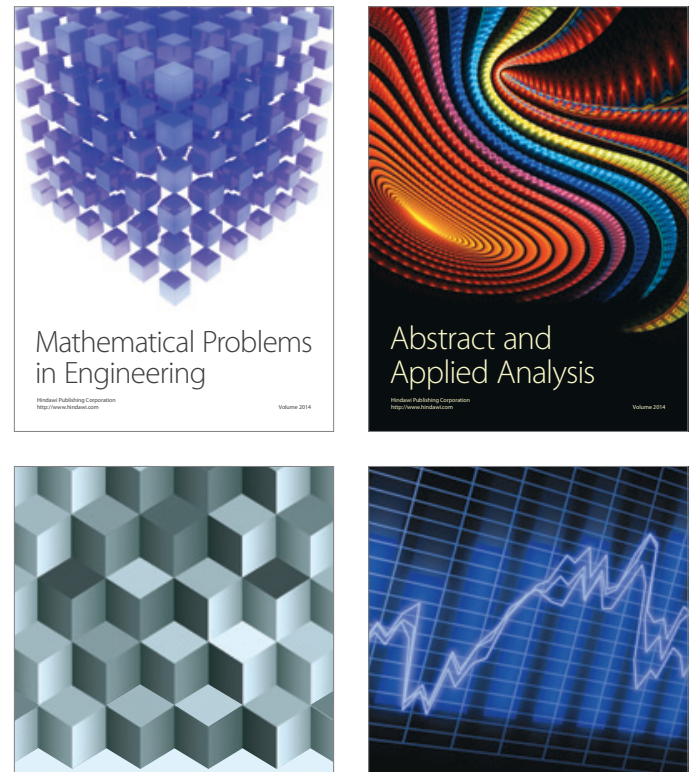

Journal of

Function Spaces

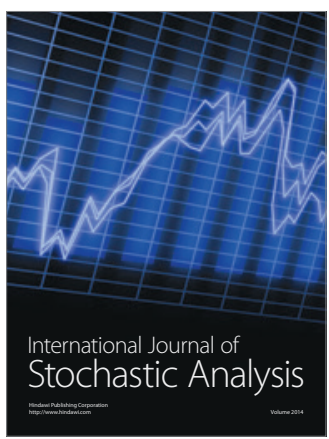

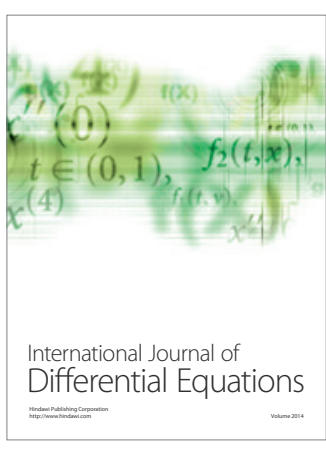
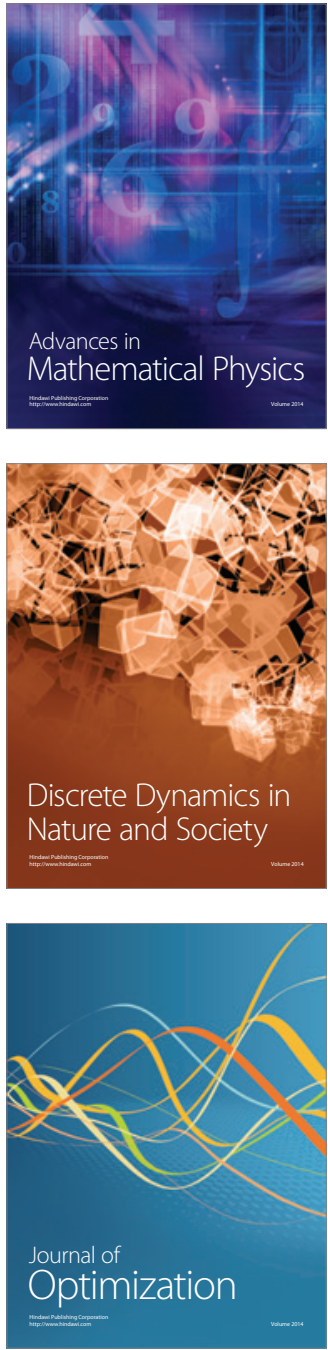